Aldridge and co-workers showed that the Ge-Ge bond in the digermavinylidene 5 is of typical length for a double bond and the coordination environment of the disubstituted germanium atom is perfectly planar. In the solid state, the germanium vinylidene species $\mathbf{5}$ is free of any external base, but features a weak interaction with the peripheral aryl groups of the diazaboryl ligands instead. The frontier orbitals of $\mathbf{5}$, however, are only mildly perturbed and resemble those of the parent $\mathbf{V}_{\mathrm{GeGe}}$ according to theoretical calculations.

By carrying out ${ }^{1} \mathrm{H}$ NMR analysis in solution, the team observed a rapid exchange process involving the aryl groups coordinating to the vacant orbital at the electron deficient germanium centre, as seen in the solid state. The activation energy for the process was estimated to an upper limit of $43 \mathrm{~kJ} \mathrm{~mol}^{-1}$ by variable temperature NMR, and the team speculates that this barrier may be associated to the 1,2-exchange of the diazaboryl ligands through a mono-bridged derivative of type $\mathbf{M}_{\text {GeGe }}$.

The unique base-free vinylidene analogue 5 will now undoubtedly be investigated with regards to its reactivity towards various reagents that exploit its high degree of functionality. On top of the double bond and the nucleophilic lone pair, the inherently electrophilic vacant $p$ orbital offers one additional handle to transform 5 into various extended systems/materials, which may retain the semiconducting element germanium in a low oxidation state as key component. Moreover, compounds such as $\mathbf{5}$ provide the urgently needed tools for the further development of the chemistry of heavier unsaturated main group species from purely curiosity-driven research into a more function-oriented direction.

For now, Aldridge and his team have given us a first exciting glimpse into the fundamental chemistry of base-free heavier vinylidenes ${ }^{10}$. When viewed together with previous results on base-coordinated species $^{7-9}$, it is clear that much more is to be expected from this unprecedented structural motif.

David Scheschkewitz holds the Chair in General and Inorganic Chemistry at Saarland University,
66123 Saarbrücken, Germany.

e-mail: Scheschkewitz@mx.uni-saarland.de

References

1. Esteban, S. J. Chem. Educ. 85, 1201-1203 (2008).

2. Breidung J. et al. Angew, Chem. Int. Ed. Engl. 36, 1983-1985 (1997).

3. Bruneau, C. \& Dixneuf, P. H. Angew. Chem. Int. Ed. 45, 2176-2203 (2006).

4. Damrauer, R. \& Noble, A. L. Organometallics 27, 1707-1715 (2008).

5. Stender, M., Phillips, A. D., Wright, R. J. \& Power, P. P. Angew. Chem. Int. Ed. 41, 1785-1787 (2002).

6. Jana, A., Huch, V. \& Scheschkewitz, D. Angew. Chem. Int. Ed. 52, 12179-12182 (2013)

7. Jana, A., Majumdar, M., Huch, V., Zimmer, M. \& Scheschkewitz, D. Dalton Trans. 43, 5175-5181 (2014).

8. Ghana, P., Arz, M. I., Das, U., Schnakenburg, G. \& Filippou, A. C. Angew. Chem. Int. Ed. 54, 9980-9985 (2015).

9. Jana, A., Omlor, I., Huch, V., Rzepa, H. S. \& Scheschkewitz, D. Angew. Chem. Int. Ed. 53, 9953-9956 (2014).

10. Rit, A., Campos, J., Niu, H. \& Aldridge, S. Nat. Chem. 8, 1022-1026 (2016)

11. Segawa, Y., Yamashita, M. \& Nozaki, K. Science 314, 113-115 (2006). 12. Green, S. P., Jones, C. \& Stasch, A. Science 318, 1754-1757 (2007). 13. Pu, L. et al. J. Am. Chem. Soc. 125, 11626-11636 (2003).

\section{Correction}

In the News \& Views 'A sweet vaccine' (Nature Chemistry 8, 201-202; 2016), an in-house error meant that Ben Davis was twice referred to as Ben Davies. This error has been corrected after print 10 October 2016.

\title{
ACTINIDE CHEMISTRY
}

\section{New compounds on the Bk menu}

For $f$-block elements like uranium, there is stabilizing coordination chemistry that can be used to demonstrate how the element reacts and forms bonds, but some of the more exotic elements in the $f$-block have not been isolated within compounds in this way. Complexes of plutonium, americium, curium and californium have provided spectroscopic insights into the interesting electronic structure of the actinides, but the gap between them - berkelium leaves questions to be answered about the factors controlling their properties. Now, a group of researchers led by Jenifer Braley and Thomas Albrecht-Schmitt at the Colorado School of Mines and Florida State University, respectively, have isolated and characterized the first complexes of berkelium(III) (Science 353, aaf3762; 2016).

The only available isotope of berkelium is ${ }^{249} \mathrm{Bk}$, which has a half-life of only 320 days. However, Braley, Albrecht-Schmitt and co-workers developed procedures for its rapid precipitation and metallation with dipicolinic acid. The resulting complexes were characterized by $\mathrm{X}$-ray diffraction, revealing $D_{3}$ symmetric nine-coordinate

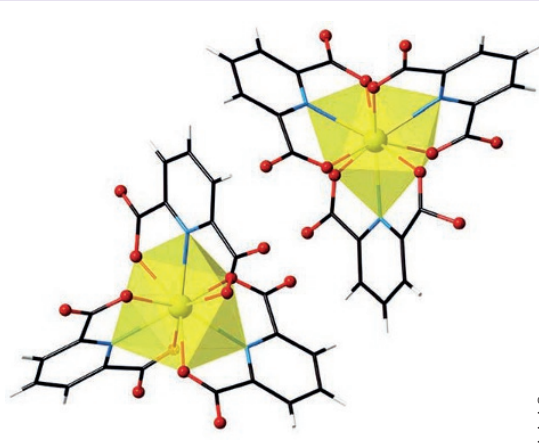

unusually high degree of covalency in the Bk-ligand bonds. These characteristics make berkelium unique among actinides.

Magnetic and theoretical analyses were carried out and comparisons between berkelium and other superheavy elements made. Despite similar electronic configurations to terbium, the electronic properties of these complexes are distinct, with spin-orbit coupling in Bk(III) dominating the ligand-field effects. The ligand-field effects of the berkelium compounds studied were observed to be similar to those of curium, but although spin-orbit coupling also dictates the electronic structure of $\mathrm{Cf}(\mathrm{III})$, ligand-field splitting in $\mathrm{Cf}(\mathrm{III})$ compounds is much greater than in those of Bk(III). This work emphasizes that spin-orbit coupling is a dominating factor in determining the ground-state structures of late actinide complexes, which may well inform the ways in which other elements from this part of the periodic table are not only characterized, but perhaps stabilized for similar studies.

MARSHALL BRENNAN 